\title{
Alpha-Stim Cranial Electrotherapy Stimulation (CES) for Anxiety Treatment: Outcomes in a United Kingdom (UK) Primary Care Practice
}

\author{
Chris Griffiths ${ }^{1 *}$, Chloe Leathlean1, David Smart², Azhar Zafar², Cara-Leigh Hall², Sarah Deeks² \\ ${ }^{1}$ Innovation and Research Department, Northamptonshire Healthcare NHS Foundation Trust (NHFT), Northampton, UK \\ ${ }^{2}$ General Practice Alliance (GPA), Northampton, UK \\ Email: *chris.griffiths@nhft.nhs.uk
}

How to cite this paper: Griffiths, C., Leathlean, C., Smart, D., Zafar, A., Hall, C.-L. and Deeks, S. (2021) Alpha-Stim Cranial Electrotherapy Stimulation (CES) for Anxiety Treatment: Outcomes in a United Kingdom (UK) Primary Care Practice. Open Journal of Psychiatry, 11, 186-201.

https://doi.org/10.4236/ojpsych.2021.113015

Received: June 11, 2021

Accepted: July 16, 2021

Published: July 19, 2021

Copyright $\odot 2021$ by author(s) and Scientific Research Publishing Inc. This work is licensed under the Creative Commons Attribution International License (CC BY 4.0).

http://creativecommons.org/licenses/by/4.0/

\begin{abstract}
Background: Anxiety disorders are the most common mental disorders, typically treated with psychotherapy and medication. These treatments are not suitable for, acceptable to, or effective for everyone. Alpha-Stim AID is a Cranial Electrotherapy Stimulation (CES) treatment with evidence of effectiveness in treating anxiety disorders. Objective: The aim of this paper is to present outcomes on anxiety, depression, and quality of life of Alpha-Stim use in primary care patients in the United Kingdom's (UK) National Health Service (NHS) who reported symptoms of anxiety. Methods: Open label patient cohort design. Self-report measures: PHQ-9 (depression), GAD-7 (anxiety) and EQ-5D-5L (health related quality of life). Twenty-three patients with symptoms of anxiety completed a six-week course of Alpha-Stim intervention. Results: Reliable improvement and remission rates respectively were $60.9 \%$ and $17.4 \%$ for the GAD-7; $42.9 \%$ and $22.7 \%$ for the PHQ-9. Significant improvement and medium/large effect sizes ( $\mathrm{n} 2=0.59$ and 0.56 respectively). EQ-5D-5L results showed significant improvements in quality of life. Perceived quality of life doubled with an improvement of 0.36 on the health index score, this intervention adds 3.64 Quality Adjusted Life Years (QALYs). Limitations: The study was not an RCT, there was no control group. Conclusions: Alpha-Stim AID CES can be delivered through a UK primary care practice, and can have a significant impact on symptoms of anxiety and depression, and improve quality of life in primary care patients with anxiety symptoms. Further feasibility studies in primary care and sufficiently powered RCT are required.
\end{abstract}

\section{Keywords}

Alpha-Stim, Primary Care, Cranial Electrotherapy Stimulation, Service 
Delivery, Anxiety, Depression, Quality of Life, Social Prescription, Co-Morbidity, GAD

\section{Introduction}

Anxiety or Generalized Anxiety Disorder (GAD) is characterized by feelings of unease, such as worry or fear, that persist for several months, resulting in distress or impairment in personal, social, educational, occupational, or other functioning areas [1]. Anxiety disorders (GAD, phobias, panic disorder) are the most common mental disorders, with lifetime prevalence rates in the general population ranging from $13.6 \%$ to $28.8 \%$ [2] [3]. GAD has a lifetime population prevalence of $3.7 \%$ and a 12 -month prevalence of $1.8 \%$ [4]. Psychiatric co-morbidities with GAD are common; this may complicate treatment and thus result in poorer treatment outcomes [5]. GAD patients use more health care resources than the general population and loss of functioning may affect their economic contribution; the costs of GAD may be comparable to Major Depressive Disorder (MDD) [5].

A systematic review and meta-analysis found that the prevalence of anxiety in the general population during the COVID-19 pandemic could be three times higher [6]; factors contributing to this include stress connected with work or job security, economic impact, loss of social connections, loss of meaningful activity, and restrictions on life [6] [7]. Many healthcare workers have experienced increased levels of anxiety due to COVID-19 [7]. Anxiety is one of the reported symptoms of long COVID [8] [9] [10], defined as symptoms during or following COVID-19 infection that continue for over 12 weeks and not explained by another diagnosis [11]. Long term effect of COVID-19 on anxiety prevalence is unknown, but COVID-19 risk and societal restrictions to prevent spread are likely to remain for a number of years.

In England and Wales, National Institute for Health and Care (NICE) guidance for management of GAD is a three-step process, step 1: identify and communicate the diagnosis and provide education and active monitoring in primary care; step 2: individual non-facilitated self-help, individual guided self-help, or psychoeducational groups (choice guided by patient preference); step 3: an individual high-intensity psychological intervention or drug treatment (choice guided by patient preference) [12]. There can be a significant wait for individual highintensity psychological intervention, and so medication may be given in advance of psychotherapy. Some people in contact with secondary mental health services are deemed not suitable for individual high-intensity psychological intervention community services, with multi-professional interventions delivered by secondary mental health care services [13].

One-to-one psychotherapy can be effective for anxiety but is costly and lengthy, with non-response rates of $60 \%-66 \%$ [14] [15]. Medication treatments for anxiety disorders include Selective Serotonin Reuptake Inhibitors (SSRIs), Sero- 
tonin Norepinephrine Reuptake Inhibitors (SNRIs), benzodiazepines, buspirone, and Tricyclic Antidepressants (TCAs) [16]. These medications can reduce symptoms of anxiety; however, not all people respond, and patient acceptability and compliance are often compromised due to adverse effects, including weight gain, increased rates of falls, increased fracture rates, gastrointestinal and sexual difficulties, insomnia, and severe headaches [17] [18] [19]. In some cases, withdrawal effects can be long-lasting and severe [20]. Use can result in increased (2.8 times) suicide risk [21]. High prescription rates are largely explicable by chronic usage, which is partially explained by patient-reported withdrawal difficulties [22]. Due to dependence and withdrawal issues, benzodiazepine is only recommended for severe anxiety and only short term prescription (two to four weeks) [23]. Some people do not respond well to either drugs or psychotherapy, and so it is important to offer alternative treatment options enhancing patient choice of treatment.

One such alternative is the Alpha-Stim AID device. Alpha-Stim Cranial Electrotherapy Stimulation (CES) uses very low voltage current to induce changes to electrical activity of the brain, from stressful (beta and delta) frequencies to more relaxing (alpha) frequencies [24]. This is delivered by the patient using a mobile phone-sized device and connected via soft pad clips to the earlobes, for up to an hour a day. It is easy to use, and is Conformitè Europëenne (CE) marked for the intended purpose. Following the completion of an intervention, Alpha-Stim devices may be returned and reused by another patient. NICE states that based on

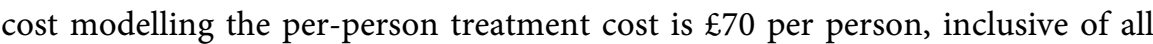
staff and ad hoc costs [25]. Alpha-Stim is safe, with NICE confirming people using Alpha-Stim AID have a low risk of side effects [25].

The Alpha-Stim has been the subject of RCTs in the United States that have demonstrated the efficacy of the treatment versus a sham device [26]. A systematic review identified five Randomised Controlled Trials (RCTs) with a total of 198 participants. The review found that it is an established and safe (does not cause serious adverse events) form of low current electrical neuromodulation that improves anxiety and depression symptoms over 6 weeks' treatment in people experiencing anxiety with depression [27]. A study set in an NHS Improving Access to Psychology Treatment (IAPT) service with 161 patients diagnosed with GAD showed the Alpha-Stim to be a clinical and cost-effective anxiety disorder treatment option [28]. There was a cost-saving of $£ 540.88$ per patient compared to Cognitive Behavioural Therapy (CBT), 44.7\% achieved anxiety remission at 12 weeks and $47.8 \%$ at 24 -week follow-up, and over $80 \%$ returned the device to the provider when finished the course of treatment [28]. In addition, scores of self-rated depression significantly reduced at week 12 , but the result was not significant at week 24 . Although this study was not an RCT, it showed that Alpha-Stim can be delivered through NHS IAPT services, is acceptable to patients and patients will conform to the required protocol. Very little is documented on the device's impact on quality of life [25], only two studies to date have reported Alpha-Stim associated improvements on the quality of life: EQ-5D-5L [28] and WHOQOL_BREF (WHO Quality of Life Questionnaire) [29]. 
There is research evidence for the effectiveness of CES in the treatment of anxiety from research trials; however, there is a lack of information on outcomes from delivering through primary care clinical practice. NICE guidelines state that Alpha-Stim AID shows "promise" for managing anxiety disorders; however, due to concern over insufficient good-quality evidence, has not to date adopted the intervention for routine support [25]. Prior to adoption, the guideline recommends the collection of real-world data on the use of Alpha-Stim AID to better understand issues around people's treatment preferences, treatment completion rates, short and long term efficacy as well as the impact on quality of life [25]. Implementation studies are recommended in healthcare settings to check that the efficacy seen in RCTs is translated into routine clinical practice [30] [31]. This is the first study to report feasibility and outcomes data from a service delivering Alpha-Stim for anxiety in primary care practice in the UK.

\section{Methods}

(1) Design

An open label patient cohort design. The project was approved by the NHS primary care provider consortium.

(2) Setting

UK National Health Service (NHS) primary care service in England.

(3) Inclusion/Exclusion Criteria

Inclusion:

1) 18 years or over

2) Signed consent form

3) Symptoms of anxiety or GAD-7 score of 8 or above

Exclusion:

1) Lack of capacity to consent

2) Experience of seizures

3) Have a pace maker of any other implanted electrical device

(4) Intervention

Electrical current is delivered by Alpha-Stim AID (CE marked medical device) $(0.5 \mathrm{~Hz}, 100-500 \mu \mathrm{A}, 50 \%$ duty cycle, biphasic asymmetrical rectangular waves), a mobile phone sized device delivering small electric currents via soft pad conducting clips to the earlobes. The person wears the device via lanyard hung around their neck, enabling light activities to be performed whilst in use. All participants were recommended 60 min per day of Alpha-Stim CES treatment at a current of one hundred micro amps (level 1,2 bars on the device display), 7 days per week for 6 consecutive weeks. Patients were given printed instructions and shown how it works. Support was provided if required. Patients remained on any physical or medical health medication they were currently taking.

(5) Measures

The measures were collected prior to treatment, mid intervention at week three and shortly following end of treatment at week six. The GAD-7 is a self-report measure of GAD [32]. A score that is rated as severe on this scale is $16-20$. The 
GAD-7 has good sensitivity and specificity for GAD and is moderately good at screening three other anxiety disorders: panic disorder, social anxiety disorder, and Post-Traumatic Stress Disorder (PTSD) [33]. It has good internal consistency: Cronbach's a 0.92 [34]. GAD-7 remission is defined as 7 points or less, reliable improvement is defined as a reduction in 5 or more points from baseline and recovery is defined as both reliable improvement and remission achievement.

Personal Health Questionnaire, 9-item (PHQ-9) a 9-item self-rated measure of the severity of depression symptoms [35]. The PHQ-9 has good sensitivity and specificity for major depression; it has good internal consistency: Cronbach's a $[0.89,35]$. A score that is rated as severe on this scale is 20 and over. PHQ-9 remission is defined as a score of 9 or less, reliable improvement is a reduction in 6 points from baseline, and recovery is defined as both reliable improvement and remission being achieved.

EQ-5D-5L is a five item self-rated measure of health related quality of life. It is a standardised measure of health status developed by EuroQol group with the aim to provide a simple, standardised measure of health for clinical appraisal [36] [37]. The descriptive systems comprises of five dimensions (mobility, self-care, usual activities, pain/discomfort, anxiety/depression) each of which are measured within 5 levels (no problems, slight problems, moderate problems, severe problems and extreme problems). The digits from the five dimensions are combined to create a five-digit number describing a participants' holistic health state. The EQ-5D-5L is a validated, generic, preference-based measure of health status, widely used in national health surveys in the UK and clinical trials of mental health interventions [38] [39]. It is recommended by NICE to estimate health state utility weights for quality-adjusted life year [21]. EQ-5D-5L demonstrates good construct validity and is sensitive to changes in in patients with depression and anxiety [40].

(6) Medical Records

Demographic information (gender, age at admission and diagnosis) were extracted from clinical records containing routinely collected data. Analysis was conducted using an anonymised database.

(7) Process to Treatment

Patients were referred to their Social Prescribing Link worker (SPLW) by their GP, who in turn referred them on to the lead SPLW for inclusion in this project. Patients were selected if they met inclusion criteria and were provided with information about the treatment and evaluation, informed consent was sought and required to begin treatment; patients could withdraw consent at any point without need for providing a reason. Figure 1 shows flow into the study.

(8) Analysis

Data were analysed using SPSS statistics software package. Data screening confirmed the dataset met all the requirements of the general linear model with the exception of baseline GAD-7 and PHQ-9 being not normally distributed; however, it is common place for clinical measures at baseline to be negatively 


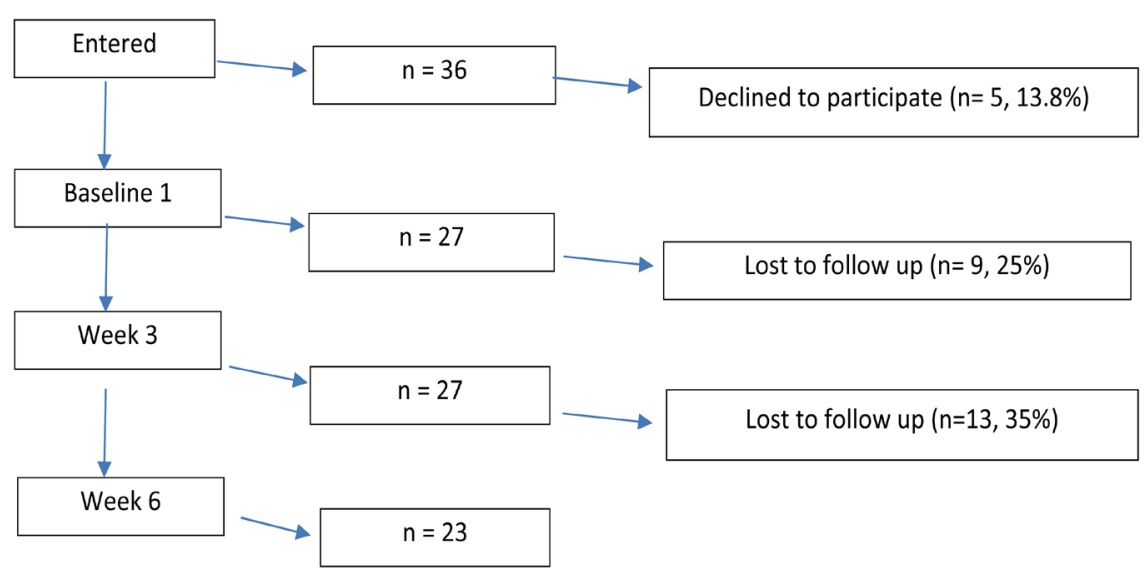

Figure 1. Flow into study.

skewed from a clinical intervention sample [41]. Following descriptive analysis, one-way repeated measures ANOVA were conducted to determine whether there were statistically significant improvements for the mental health assessments between baseline, week 3 and week 6 following the intervention. The EQ-5D-5L individual dimensions were primarily analysed using descriptive statistics. One-way repeated measures ANOVA were administered to assess whether the improvements observed in each dimension were significant. The average digit calculated within each dimension was combined at baseline and post intervention to create two comparative five-digit health states. These digits were converted into the corresponding holistic health index scores to calculate quality-adjusted life years (QALYs). A one-way repeated measure ANOVA was conducted on each participant's converted health index score at the three time points to assess significant improvement. Finally, ANCOVA was utilized to test whether CES usage acted as a significant covariate of improvement observed in GAD-7, PHQ-9 and health.

\section{Results}

Of the 36 participants recruited, 23 (63.89\%) completed 6 weeks of treatment and post intervention mental health assessments. The sample comprised of 29 (80.6\%) females and seven males (19.4\%). Prior to week 1 five participants disengaged from the research. Of the given reasons, one stated extreme external pressure (2.7\%), 1 stated severe headache (2.7\%), 1 stated extreme anxiety (2.7\%) and 2 declined for unknown reasons (5.4\%). Following baseline measure, four participants withdrew; two (5.6\%) stated they experienced anxiety regarding the device; $1(2.7 \%)$ withdrew due to concern about medical history and one $(2.7 \%)$ for further unknown reasons. Prior to week 6, four participants were lost to follow up; one stated extreme headaches (2.7\%), one felt unable to continue due to lack of coping following the bereavement of family member $(2.7 \%)$, the remaining two did not stipulate a reason for disengagement (5.5\%). As illustrated by Table 1, participant mean baseline scores were in the severe range for GAD and 
Table 1. Baseline characteristics of participants $(n=36)$.

\begin{tabular}{cc}
\hline Variable & Age, Mean \pm SD (Min-Max $) \%$ \\
\hline Age & $18-65$ \\
Health Index & $0.29 \pm 0.32(-0.28-0.82)$ \\
GAD7 & $17.93 \pm 3.41(11-21)$ \\
PHQ9 & $19.00 \pm 4.1(7-25)$ \\
Female & $29(80.6 \%)$ \\
\hline
\end{tabular}

moderately severe range for depression [32] [35]. Baseline EQ-5D-5L crosswalk data values indicated participants had a holistic health index of $0.29(\max =1)$.

Table 2 shows the proportion of participants who reach remission, reliable improvement and recovery for GAD-7 and PHQ-9 at week 3 and week 6 following the intervention. By week three, 2 (7.7\%) of participants achieved both remission and recovery. The proportion of participants that achieved reliable improvement was $9(34.6 \%)$ and $14(60.9 \%)$ at 3 and 6 weeks respectively. No participants reported deterioration in either GAD-7 or PHQ-9 over the intervention.

Prior to statistical analysis, data screening was conducted to evaluate whether the dataset met the requirements of the general linear model. One-way repeated measures ANOVA was conducted to determine whether there were statistically significant differences in GAD-7 scores over the course of the 6 week alpha stim intervention. There were no outliers in the data, as assessed by inspection of a boxplot. The Shapiro Wilk's test assessed both the GAD-7 and PHQ-9 baseline measures to not be normally distributed $(p<0.05)$. However, it is common for clinical measures at baseline to be negatively skewed from a clinical intervention sample [41]. GAD-7 and PHQ-9 scores were normally distributed at week 3 and week 6, as assessed by Shapiro-Wilk's tests ( $p>0.05$ ). Mauchly's test of sphericity indicated that the assumption of sphericity had not been violated, $\chi^{2}(2)=0.15$, $\mathrm{p}=0.93$. The reduction in GAD-7 scores were statistically significant, $\mathrm{F}(2,44)=$ $30.35, \mathrm{p}<0.001$, a large effect size was observed partial $\mathrm{n} 2=0.59$. Thus, $59 \%$ of the improvement in GAD score was attributed to the intervention. Post hoc analysis with a Bonferroni adjustment revealed that there was a decrease in GAD-7 score from baseline $(\mathrm{M}=17.77, \mathrm{SD}=3.37)$ to week $3(\mathrm{M}=13.68, \mathrm{SD}=$ 4.43), a statistically significant mean decrease of 4.09, 95\% CI [2.1, 6.09], p < 0.001 ; and from baseline to week $6(\mathrm{M}=11.95, \mathrm{SD}=4.29)$.

In comparison to GAD-7, a lower percentage of participants achieved reliable PHQ-9 improvement by week 3 and week 6, yet more participants achieved remission at week 3 and 6 . By the end of the intervention an equivalent percentage of recovery was observed for PHQ-9 and GAD-7. Spearman rank-order correlation indicated a statistically significant correlation between PHQ-9 and GAD-7 improvement over time, $\mathrm{r}_{\mathrm{s}}=0.63, \mathrm{p}=0.001$

The PHQ-9 effects were similar to GAD-7. Mauchly's test of sphericity indicated that the assumption of sphericity had not been violated, $\chi^{2}(2)=2.04, p=$ 
Table 2. Remission, reliable improvement and recovery.

\begin{tabular}{ccccccc}
\hline & \multicolumn{3}{c}{3 weeks } & \multicolumn{3}{c}{6 weeks } \\
\cline { 2 - 7 } Outcome & Remission & $\begin{array}{c}\text { Reliable } \\
\text { improve }\end{array}$ & Recovery & Remission & $\begin{array}{c}\text { Reliable } \\
\text { improve }\end{array}$ & Recovery \\
\cline { 2 - 7 } & $\mathrm{n}=26(\%)$ & $\mathrm{n}=26(\%)$ & $\mathrm{n}=26(\%)$ & $\mathrm{n}=22(\%)$ & $\mathrm{n}(\%)$ & $\mathrm{n}(\%)$ \\
\hline GAD-7 & $2(7.7 \%)$ & $9(34.6 \%)$ & $\begin{array}{c}2(7.7 \%) \\
\mathrm{n}=26\end{array}$ & $4(17.4 \%)^{*}$ & $14(60.9 \%)^{*}$ & $4(17.4 \%)^{*}$ \\
& & & $1(3.8 \%)$ & $5(22.7 \%)$ & $12(42.9 \%)$ & $4(14.3 \%)$ \\
PHQ-9 & $4(15.4 \%)$ & $7(26.9 \%)$ & $\mathrm{n}=26$ & & & \\
& & & $\begin{array}{c}1(3.8 \%) \\
\mathrm{n}=26\end{array}$ & $3(13.6 \%)$ & $12(54.5 \%)$ & $3(13.6 \%)$ \\
\hline
\end{tabular}

${ }^{*} \mathrm{n}=23$.

0.36. The reduction in PHQ-9 scores were statistically significant at all time points during the alpha stim intervention, $\mathrm{F}(2,40)=25.41, \mathrm{p}<0.001$, partial $\mathrm{n} 2$ $=0.56$. The large effect size confers $56 \%$ of the improvement in PHQ-9 was accounted for by the intervention. Post hoc analysis with Bonferroni adjustment revealed that there was a decrease in PHQ-9 score from baseline $(\mathrm{M}=18.52$, SD $=4.03)$ to week three $(\mathrm{M}=15.24, \mathrm{SD}=5.20)$, a statistically significant mean decrease of 3.29 95\% CI [1.53, 5.04], p < 0.001; Similarly, a decrease in PHQ-9 score is observed between baseline and week $6(\mathrm{M}=12.81, \mathrm{SD}=5.08)$, a statistically significant decrease of 5.71, 95\% CI [3.37, 8.06], $\mathrm{p}<0.001$.

Table 3 illustrates the data collected from the EQ-5D-5L tool at baseline and week 3 and 6 following the intervention. From the table, the data depicts the intervention did not play a large role in improving mobility. At week $6,8.3 \%$ of participants reported the experience of severe/extreme mobility issues, compared to $18.5 \%$ at baseline, illustrating an improvement in $10.2 \%$ of participants. A greater improvement was observed in the selfcare dimension in which at baseline $11.1 \%$ of participants reported they had severe problems washing or dressing themselves, whereas at week 3 and week 6 this severity of dimension was not reported by any participants. Furthermore, at baseline $29.6 \%$ of participants reported they had no problems washing or dressing themselves, whereas by week 6 this was reported by $45.8 \%$ of participants. Following the intervention a similar degree of improvement was observed in the ability to engage in usual activities dimension. Whereas at baseline $0 \%$ of participants reported being able to get on with usual activities without problems, by the end of week $6,8.3 \%$ of participants had acquired this level. A large improvement (23.1\%) was observed in participants at baseline who had extreme/severe inability to conduct their usual activities $(48.1 \%)$ to week six (25.0\%). Minimal improvement was found in the pain/discomfort dimension. The greatest improvement was observed in the depression/anxiety dimension. At baseline $40.7 \%$ of participants stated they were extremely anxious or depressed; whereas at week 6 no participants reported the extreme level. No participants reported being totally free from anxiety or depression, but there was an improvement of $16.7 \%$ of participants who reported 
Table 3. Heath profile: percentage of participants that reported levels 1 to 5 on EQ-5D-5L by dimension and time.

\begin{tabular}{|c|c|c|c|c|}
\hline \multicolumn{2}{|c|}{ EQ-5D Dimension } & Baseline & Week 3 & Week 6 \\
\hline \multirow{8}{*}{ Mobility } & Level 1 & $48.1 \%$ & $44.4 \%$ & $41.7 \%$ \\
\hline & Level 2 & $14.8 \%$ & $18.5 \%$ & $29.2 \%$ \\
\hline & Level 3 & $18.5 \%$ & $22.2 \%$ & $20.8 \%$ \\
\hline & Level 4 & $14.8 \%$ & $11.1 \%$ & $8.3 \%$ \\
\hline & Level 5 & $3.7 \%$ & $3.7 \%$ & $0.0 \%$ \\
\hline & No problems & $48.1 \%$ & $44.4 \%$ & $41.7 \%$ \\
\hline & Slight-moderate & $33.3 \%$ & $40.7 \%$ & $50.0 \%$ \\
\hline & Severe-extreme & $18.5 \%$ & $14.8 \%$ & $8.3 \%$ \\
\hline \multirow{8}{*}{ Self care } & Level 1 & $29.6 \%$ & $33.3 \%$ & $45.8 \%$ \\
\hline & Level 2 & $29.6 \%$ & $29.6 \%$ & $33.3 \%$ \\
\hline & Level 3 & $29.6 \%$ & $37.0 \%$ & $20.8 \%$ \\
\hline & Level 4 & $11.1 \%$ & $0.0 \%$ & $0.0 \%$ \\
\hline & Level 5 & $0.0 \%$ & $0.0 \%$ & $0.0 \%$ \\
\hline & No problems & $29.6 \%$ & $33.3 \%$ & $45.8 \%$ \\
\hline & Slight-moderate & $59.2 \%$ & $66.6 \%$ & $54.1 \%$ \\
\hline & Severe-extreme & $11.1 \%$ & $0.0 \%$ & $0.0 \%$ \\
\hline \multirow{8}{*}{ Usual activity } & Level 1 & $0.0 \%$ & $3.7 \%$ & $8.3 \%$ \\
\hline & Level 2 & $7.4 \%$ & $18.5 \%$ & $33.3 \%$ \\
\hline & Level 3 & $44.4 \%$ & $44.4 \%$ & $33.3 \%$ \\
\hline & Level 4 & $25.9 \%$ & $29.6 \%$ & $20.8 \%$ \\
\hline & Level 5 & $22.2 \%$ & $3.7 \%$ & $4.2 \%$ \\
\hline & No problems & $0.0 \%$ & $3.7 \%$ & $8.3 \%$ \\
\hline & Slight-moderate & $51.8 \%$ & $62.9 \%$ & $66.6 \%$ \\
\hline & Severe-extreme & $48.1 \%$ & $33.3 \%$ & $25.0 \%$ \\
\hline \multirow{8}{*}{ Pain/discomfort } & Level 1 & $29.6 \%$ & $40.7 \%$ & $20.8 \%$ \\
\hline & Level 2 & $22.2 \%$ & $22.2 \%$ & $33.3 \%$ \\
\hline & Level 3 & $33.3 \%$ & $14.8 \%$ & $29.2 \%$ \\
\hline & Level 4 & $0.0 \%$ & $3.7 \%$ & $12.5 \%$ \\
\hline & Level 5 & $14.3 \%$ & $18.5 \%$ & $4.2 \%$ \\
\hline & No problems & $29.6 \%$ & $40.7 \%$ & $20.8 \%$ \\
\hline & Slight-moderate & $55.5 \%$ & $37.0 \%$ & $62.5 \%$ \\
\hline & Severe-extreme & $14.3 \%$ & $22.2 \%$ & $16.7 \%$ \\
\hline \multirow{8}{*}{ Anxiety/depression } & Level 1 & $0.0 \%$ & $0.0 \%$ & $0.0 \%$ \\
\hline & Level 2 & $0.0 \%$ & $14.8 \%$ & $16.7 \%$ \\
\hline & Level 3 & $25.0 \%$ & $33.3 \%$ & $33.3 \%$ \\
\hline & Level 4 & $33.3 \%$ & $37.0 \%$ & $50.0 \%$ \\
\hline & Level 5 & $40.7 \%$ & $14.8 \%$ & $0.0 \%$ \\
\hline & No problems & $0.0 \%$ & $0.0 \%$ & $0.0 \%$ \\
\hline & Slight-moderate & $25.0 \%$ & $48.1 \%$ & $50.0 \%$ \\
\hline & Severe-extreme & $74.0 \%$ & $51.8 \%$ & $50.0 \%$ \\
\hline
\end{tabular}

No problems: level 1. Slight-moderate problems: sum of level 2 and 3. Severe-extreme: sum of level 4 and 5. 
they experienced only slight anxiety/depression. Table 3 provides a summary of the statistical improvements observed in the five dimensions, across each of the five levels from baseline to week 6 following the intervention. Statistically significant improvements are observed in selfcare, ability to perform usual activities as well as experience of depression and anxiety.

Research has established a corresponding EQ-5D-5L digit health index score which is utilized to calculate Quality-Adjusted Life Years (QALYs). The dimensions given the greatest importance in the contribution to quality of life in the UK population are pain/discomfort and anxiety/depression [42]. Table 4 illustrates the descriptive data for each of the five dimensions as well as the mean health digit (calculated according to each dimension) and corresponding health index at baseline, week 3 and week 6 . The average digit in each dimension were combined at baseline and converted to a health index value of $0.357(\max =1)$, adopting the same process following the intervention the improved health digit converts to a score of 0.721 . Thus, from baseline to week 6 , quality of life doubles with an improvement of 0.36 reported. Measured across ten years, this intervention adds 3.64 QALYs.

Additionally, a one-way repeated measure ANOVA was conducted to determine whether there was a statistically significant difference in participants health index score over the course of the 6 week alpha stim intervention. There were no outliers in the data, as assessed by inspection of a boxplot. The Shapiro Wilk's test assessed the health index values to be normally distributed at baseline, week 3 and week 6 ( $p>0.05$ ). Mauchly's test of sphericity indicated that the assumption of sphericity had not been violated, $\chi^{2}(2)=0.91, p=0.374$. The improvement in health index score was statistically significant, $F(1,21)=11.40, \mathrm{p}=$ 0.003 , partial $\mathrm{n} 2=0.35$ a medium effect size. Post hoc analysis with a Bonferroni adjustment revealed there was an improvement from baseline $(\mathrm{M}=0.32, \mathrm{SD}=$ $0.31)$ to week $6(\mathrm{M}=0.51, \mathrm{SD}=0.24)$, a statistically significant mean improvement of $0.1995 \%$ CI $[0.05,0.34], \mathrm{p}=0.009$.

Table 4. Mean and standard deviation within each dimension across time with corresponding mean variation, significance and effect size.

\begin{tabular}{|c|c|c|c|c|c|c|}
\hline \multirow{2}{*}{ EQ-5D Dimension } & Baseline & Week 3 & Week 6 & \multirow{2}{*}{$\mathrm{F}$} & \multirow{2}{*}{$\mathrm{p}$} & \multirow{2}{*}{$\mathrm{n} 2$} \\
\hline & Mean (std) & Mean (std) & Mean (std) & & & \\
\hline Mobility level & $2.11(1.28)$ & $2.11(1.22)$ & $1.96(1.00)$ & 0.792 & 0.459 & 0.04 \\
\hline Selfcare level & $2.22(1.01)$ & $2.04(0.85)$ & $1.75(0.79)$ & 5.58 & $0.015^{\star}$ & 0.21 \\
\hline Usual activity level & $3.63(0.93)$ & $3.11(0.89)$ & $2.79(1.02)$ & 11.01 & $\mathrm{p}<0.001^{*}$ & 0.34 \\
\hline Pain/discomfort level & $2.48(1.34)$ & $2.37(1.52)$ & $2.46(1.10)$ & 0.69 & 0.507 & 0.03 \\
\hline Anxiety/depression level & $4.15(0.82)$ & $3.52(0.93)$ & $3.33(0.76)$ & 9.31 & $\mathrm{p}<0.001^{\star}$ & 0.31 \\
\hline Combined level health state & 22324 & 22323 & 11223 & & & \\
\hline Health index score & 0.357 & 0.57 & 0.721 & & & \\
\hline
\end{tabular}

* Significant at $\mathrm{p}<0.05$ level. Each dimension is scored between 1 -no problem to 5-extreme problem. Health state is defined by combing the level from each of the 5 dimensions into a single health digit. The combined health digit converts into health index score out of 1 . 
As stipulated within the procedures, participants were required to use the Alpha-Stim intervention daily. Data from the participant usage questionnaire indicated that $29.6 \%$ of participants used the device daily through the 6 week intervention, $48.1 \%$ reported using the device "virtually" everyday, $18.5 \%$ used the device most days and $3.7 \%$ of participants used the device half of the days. These categories were analysed as a covariate between PHQ-9 and GAD-7 improvement. The ANCOVA results were non-significant in both cases, indicating that failure to adhere to daily treatment did not have a significant detrimental impact on improvement.

\section{Discussion}

The results show that Alpha-Stim CES can be delivered in a UK based primary care clinical service and can be effective in reducing anxiety. The outcomes are comparable to published RCTs and service data. In moderate to severe anxiety patients, $17.4 \%$ of patients achieved remission and $60.9 \%$ showed reliable improvement in their self-rated anxiety symptoms with 6 weeks of Alpha-Stim CES treatment. Morriss et al. (2019) reported $44.7 \%$ remission and $63.4 \%$ reliable improvement rate [28]. Although similar reliable improvements were observed, the current study's higher GAD-7 baseline score may account for the lower remission rates. This present study shows that use of the device may lead to rapid improvement, $34.6 \%$ of participants show reliable improvement within 3 weeks.

In terms of the depression results, remission and reliable improvement were $22.7 \%$ and $42.9 \%$ respectively following the six week intervention. Morriss et al. reported a similar reliable improvement rate (47.2\%) and a higher remission rate (45.3\%) [28]. Baseline demographics revealed the current sample had more severe symptoms as illustrated by PHQ-9 (mean $=19$ verses 16.07). As with anxiety, reliable improvement can be achieved quickly, the current study indicated $26.9 \%$ of participants made reliable PHQ-9 improvement by week three.

The current paper is the first to publish a detailed analysis on the impact of Alpha-Stim intervention on quality of life using the EQ-5D-5L. The results indicated a positive outcome on four of the five dimensions measured by the EQ-5D-5L. Of these improvements the dimensions of self-care, ability perform usual activities and anxiety/depression were statistically significant. The largest effect sizes were observed in ability to perform usual activities and the anxiety/depression dimension. This indicates the positive impact of Alpha-Stim on the real world functioning and wellbeing of patients.

The only EQ-5D-5L dimension that failed to indicate improvement was the pain/discomfort dimension. It is perhaps expected that this is the case as pain and discomfort are often due to long term chronic physical health problems. Meta-analysis of research data indicates use of CES for pain conditions (such as fibromyalgia, headache, painful degenerative join disease, low back pain, musculoskeletal pain, and chronic neuromuscular pain) have mixed results [27].

The EQ-5D-5L health index conversion scores indicated that the quality of life 
improved following the six week intervention The 0.36 improvement is equivalent to 3.64 QALYs (life year gains) across the span of 10 years. The cost per QALY threshold stipulated by NICE for England and Wales ranges between $£ 20,000$ and $£ 30,000$; NICE reported cost modelling estimated a per-person treatment cost of $£ 70$ for a 10 week course of Alpha-Stim and inclusive of all staff and ad hoc costs [25]. Thus, the intervention is highly cost effective, as price per QALY is well below the stipulated threshold.

The treatment was acceptable to most patients, most used as the device as instructed and returned it following use. Results indicate that this approach is effective in targeting anxiety irrespective of the existence of co-morbid illness as well depression. The intervention's success indicates the effectiveness of NHS delivered CES and the need to increase availability to enable more people with anxiety to potentially benefit. This intervention may offer hope to the many people with complex mental and physical health histories who experience symptoms of anxiety or depression that have failed to respond to medication or psychotherapy or who find medication side effects unacceptable. Cost-benefit analysis is required to understand the potential savings that could be derived by the wider implementation of Alpha-stim for people with anxiety. Further research is required to investigate why some people respond and others do not: what factors determine response.

\section{Limitations}

This was not a randomised controlled trial; there was no control group. Treatment was open label and adjunct to any existing anxiety or other treatments. There was no follow-up data collection point beyond end of treatment, and thus long-term effects could not be reported. The sample was over-represented by females $(80 \%)$. An attrition rate of $36 \%$ was observed, with 36 participants entering the study and 23 completing the 6 week intervention. A number of participants disengaged from the research without explanation and therefore it is unclear if any participants disengaged for positive reasons, such as they no longer required treatment due to improvement. In the current research, CES treatment dosage was not individualised per participant; for example, participants may benefit from distinct waveforms, intensity and stimulus location [42], but it is not known if this is the case. The devices were not locked and therefore some participants may have used a higher than recommended dosage.

\section{Conclusion}

Further work is needed to define the role of CES in an anxiety treatment pathway. This work needs to understand when it is best to offer CES in people's experience of anxiety and when CES is a better option than other treatment options such as psychotherapy or medications. Due to low side effects, the intervention could be considered in advance of using medication. The availability of CES is currently limited. The results support a wider availability of CES as a treat- 
ment option for people with anxiety. Ideally, CES should be a treatment option freely available to people with mental health illnesses who meet the criteria for treatment rather than just those who can afford the cost of the device for themselves. There is a need for further RCTs on effectiveness for anxiety: an RCT comparing Alpha-Stim AID with individual Cognitive Behavioural Therapy (CBT), medication or both [25]. There is also a need to improve the understanding of Alpha-Stim AID's mechanism of action through studies that include a sham control [25].

\section{Key Points}

- Primary care delivered Alpha-Stim for anxiety treatment can reduce anxiety and depression symptoms and improve quality of life.

- Alpha-stim is effective irrespective of the existence of co-morbid physical or mental illness.

- Alpha-stim should be a treatment option available to people with anxiety.

\section{Acknowledgements}

The authors wish to thank the patients and staff involved and National Institute for Health Research (NIHR) Clinical Research Network (CRN) East Midlands for their financial support.

\section{Conflicts of Interest}

Authors have no conflicts of interest, and the work was not supported or funded by a company.

\section{References}

[1] Kogan, C.S., Stein, D.J., Maj, M., First, M.B., Emmelkamp, P.M. and Reed, G.M. (2016) The Classification of Anxiety and Fear-Related Disorders in the ICD-11. Depression and Anxiety, 33, 1141-1154. https://doi.org/10.1002/da.22530

[2] Kessler, R.C. and Wang, P.S. (2008) The Descriptive Epidemiology of Commonly Occurring Mental Disorders in the United States. Annual Review of Public Health, 29, 115-129. https://doi.org/10.1146/annurev.publhealth.29.020907.090847

[3] Michael, T., Zetsche, U. and Margraf, J. (2007) Epidemiology of Anxiety Disorders. Psychiatry, 6, 136-142. https://doi.org/10.1016/j.mppsy.2007.01.007

[4] Ruscio, A.M., Hallion, L.S., Lim, C.C.W., Aguilar-Gaxiola, S., Al-Hamzawi, A., Alonso, J., Andrade, L.H., Borges, G., Bromet, E.J., Bunting, B., Caldas de Almeida, J.M., Demyttenaere, K., Florescu, S., de Girolamo, G., Gureje, O., Haro, J.M., He, Y., Hinkov, H., Hu, C., de Jonge, P., Karam, E.G., Lee, S., Lepine, J.P., Levinson, D., Mneimneh, Z., Navarro-Mateu, F., Posada-Villa, J., Slade, T., Stein, D.J., Torres, Y., Uda, H., Wojtyniak, B., Kessler, R.C., Chatterji, S. and Scott, K.M. (2017) CrossSectional Comparison of the Epidemiology of DSM-5 Generalized Anxiety Disorder across the Globe. JAMA Psychiatry, 74, 465-475.

https://doi.org/10.1001/jamapsychiatry.2017.0056

[5] Revicki, D.A., Travers, K., Wyrwich, K.W., Svedsäter, H., Locklear, J., Mattera, M. S., Montgomery, S., et al. (2012) Humanistic and Economic Burden of Generalized 
Anxiety Disorder in North America and Europe. Journal of Affective Disorders, 140, 103-112. https://doi.org/10.1016/j.jad.2011.11.014

[6] Santabárbara, J., Lasheras, I., Lipnicki, D.M., Bueno-Notivol, J., Moreno, M.P., López-Antón, R., Gracia-García, P., et al. (2021) Prevalence of Anxiety in the COVID-19 Pandemic: An Updated Meta-Analysis of Community-Based Studies. Progress in Neuro-Psychopharmacology and Biological Psychiatry, 109, Article ID: 110207. https://doi.org/10.1016/j.pnpbp.2020.110207

[7] Santabárbara, J., Bueno-Notivol, J., Lipnicki, D.M., Olaya, B., Pérez-Moreno, M., Gracia-García, P., Ozamiz-Etxebarria, N., et al. (2021) Prevalence of Anxiety in Health Care Professionals during the COVID-19 Pandemic: A Rapid Systematic Review (on Published Articles in Medline) with Meta-Analysis. Progress in Neuro-Psychopharmacology and Biological Psychiatry, 107, Article ID: 110244. https://doi.org/10.1016/j.pnpbp.2020.110207

[8] Mahase, E. (2020) Covid-19: What Do We Know about "Long Covid"? BMJ, 370, m2815. https://doi.org/10.1136/bmj.m2815

[9] NHS (2021) Long-Term Effects of Coronavirus (Long COVID). https://www.nhs.uk/conditions/coronavirus-covid-19/long-term-effects-of-coronavi rus-long-covid

[10] Yelin, D., Margalit, I., Yahav, D., Runold, M. and Bruchfeld, J. (2021) Long COVID-19-It's Not over Until? Clinical Microbiology and Infection, 27, 506-508. https://doi.org/10.1016/j.cmi.2020.12.001

[11] NICE (2020) COVID-19 Guideline Scope: Management of the Long-Term Effects of COVID-19. https://www.nice.org.uk/guidance/ng188/documents/final-scope

[12] NICE (2019) Generalised Anxiety Disorder and Panic Disorder in Adults: Management. Clinical Guideline [CG113]. https://www.nice.org.uk/guidance/cg113

[13] National Collaborating Centre for Mental Health (2020) The Improving Access to Psychological Therapies (IAPT) Manual.

https://www.england.nhs.uk/wp-content/uploads/2020/05/iapt-manual-v4.pdf

[14] Gyani, A., Shafran, R., Layard, R. and Clark, D.M. (2013) Enhancing Recovery Rates: Lessons from Year One of IAPT. Behaviour Research and Therapy, 51, 597-606. https://doi.org/10.1016/j.brat.2013.06.004

[15] Griffiths, C.A. and Griffiths, L.J. (2014) Recovery and Reliable Change Rates for Patients Scoring Severe on Depression, Anxiety or Impaired Functioning in a Psychological Therapies Service: IAPT. Mental Health Review Journal, 20, 28-35. https://doi.org/10.1108/MHRJ-06-2014-0022

[16] Bespalov, A.Y., van Gaalen, M.M. and Gross, G. (2009) Antidepressant Treatment in Anxiety Disorders. In: Behavioral Neurobiology of Anxiety and Its Treatment, Springer, Berlin, 361-390. https://doi.org/10.1007/7854 20093

[17] Anderson, H.D., Pace, W.D., Libby, A.M., West, D.R. and Valuck, R.J. (2012) Rates of 5 Common Antidepressant Side Effects among New Adult and Adolescent Cases of Depression: A Retrospective US Claims Study. Clinical Therapeutics, 34, 113-123. https://doi.org/10.1016/j.clinthera.2011.11.024

[18] Gafoor, R., Booth, H.P. and Gulliford, M.C. (2018) Antidepressant Utilisation and Incidence of Weight Gain during 10 Years' Follow-Up: Population Based Cohort Study. BMJ, 361, k1951. https://doi.org/10.1136/bmj.k1951

[19] Lingam, R. and Scott, J. (2002) Treatment Non-Adherence in Affective Disorders. Acta Psychiatrica Scandinavica, 105, 164-172.

https://doi.org/10.1034/j.1600-0447.2002.1r084.x 
[20] Davies, J. and Read, J. (2019) A Systematic Review into the Incidence, Severity and Duration of Antidepressant Withdrawal Effects: Are Guidelines Evidence-Based? Addictive Behaviors, 97, 111-121. https://doi.org/10.1016/j.addbeh.2018.08.027

[21] Hengartner, M.P. and Plöderl, M. (2019) Newer-Generation Antidepressants and Suicide Risk in Randomized Controlled Trials: A Re-Analysis of the FDA Database. Psychotherapy and Psychosomatics, 88, 247-248. https://doi.org/10.1159/000501215

[22] Read, J., Gee, A., Diggle, J. and Butler, H. (2019) Staying on, and Coming off, Antidepressants: The Experiences of 752 UK Adults. Addictive Behaviors, 88, 82-85. https://doi.org/10.1016/j.addbeh.2018.08.021

[23] NICE (2019) Benzodiazepine and Z-Drug Withdrawal. https://cks.nice.org.uk/topics/benzodiazepine-z-drug-withdrawal

[24] Kennerly, R. (2004) QEEG Analysis of Cranial Electrotherapy: A Pilot Study. Journal of Neurotherapy, 8, 112-112.

[25] NICE (2021) Alpha-Stim AID for Anxiety Disorders. https://www.nice.org.uk/guidance/mtg56

[26] Barclay, T.H. and Barclay, R.D. (2014) A Clinical Trial of Cranial Electrotherapy Stimulation for Anxiety and Comorbid Depression. Journal of Affective Disorders, 164, 171-177. https://doi.org/10.1016/j.jad.2014.04.029

[27] Shekelle, P., Cook, I., Miake-Lye, I.M., Mak, S., Booth, M.S., Shanman, R. and Beroes, J.M. (2018) The Effectiveness and Risks of Cranial Electrical Stimulation for the Treatment of Pain, Depression, Anxiety, PTSD, and Insomnia: A Systematic Review. Department of Veterans Affairs (US), Washington DC. https://www.ncbi.nlm.nih.gov/books/NBK493132

[28] Morriss, R., Xydopoulos, G., Craven, M., Price, L. and Fordham, R. (2019) Clinical Effectiveness and Cost Minimisation Model of Alpha-Stim Cranial Electrotherapy Stimulation in Treatment Seeking Patients with Moderate to Severe Generalised Anxiety Disorder. Journal of Affective Disorders, 253, 426-437. https://doi.org/10.1016/j.jad.2019.04.020

[29] Hu, J. and Lu, L. (2014) A Comparative Study of Anxiety Disorders Treatment with Paroxetine in Combination with Cranial Electrotherapy Stimulation Therapy. Medical Innovation of China, 11, 80-82.

https://www.alpha-stim.com/comparative-study-anxiety-disorders-treatment-parox etine-combination-cranial-electrotherapy-stimulation-therapy

[30] Medical Research Council (MRC) (2000) A Framework for Development and Evaluation of Randomised Controlled Trials for Complex Interventions to Improve Health. Medical Research Council, London.

[31] NICE (2018) Guide to the Processes of Technology Appraisal. Guide to the Processes of Technology Appraisal. NICE, London.

[32] Spitzer, R.L., Kroenke, K., Williams, J.B. and Löwe, B. (2006) A Brief Measure for Assessing Generalized Anxiety Disorder: The GAD-7. Archives of Internal Medicine, 166, 1092-1097. https://doi.org/10.1001/archinte.166.10.1092

[33] Kroenke, K., Spitzer, R.L., Williams, J.B., Monahan, P.O. and Löwe, B. (2007) Anxiety Disorders in Primary Care: Prevalence, Impairment, Comorbidity, and Detection. Annals of Internal Medicine, 146, 317-325. https://doi.org/10.7326/0003-4819-146-5-200703060-00004

[34] Kroenke, K., Spitzer, R.L. and Williams, J.B. (2001) The PHQ-9: Validity of a Brief Depression Severity Measure. Journal of General Internal Medicine, 16, 606-613. https://doi.org/10.1046/j.1525-1497.2001.016009606.x

[35] EuroQol Group (1990) EuroQol-A New Facility for the Measurement of Health- 
Related Quality of Life. Health Policy, 16, 199-208. https://doi.org/10.1016/0168-8510(90)90421-9

[36] Van Hout, B., Janssen, M.F., Feng, Y.S., Kohlmann, T., Busschbach, J., Golicki, D., Pickard, A.S., et al. (2012) Interim Scoring for the EQ-5D-5L: Mapping the EQ-5D-5L to EQ-5D-3L Value Sets. Value in Health, 15, 708-715. https://doi.org/10.1016/j.jval.2012.02.008

[37] Brooks, R. and Group, E. (1996) EuroQol: The Current State of Play. Health Policy, 37, 53-72. https://doi.org/10.1016/0168-8510(96)00822-6

[38] Herdman, M., Gudex, C., Lloyd, A., Janssen, M.F., Kind, P., Parkin, D., Badia, X., et al. (2011) Development and Preliminary Testing of the New Five-Level Version of EQ-5D (EQ-5D-5L). Quality of Life Research, 20, 1727-1736.

https://doi.org/10.1007/s11136-011-9903-x

[39] Peasgood, T., Brazier, J. and Papaioannou, D. (2012) A Systematic Review of the Validity and Responsiveness of EQ-5D and SF-6D for Depression and Anxiety. HEDS Discussion Paper, 12-15.

[40] Pallant, J. (2016) SPSS Survival Manual. 6th Edition, McGraw-Hill Education, New York.

[41] Devlin, J.N., Shah, K.K., Feng, Y., Mulhern, B. and Hout, B. (2016) Valuing Health-Related Quality of Life: An EQ5D5L Value Set for England. Health Economics, 27, 7-22. https://doi.org/10.1002/hec.3564

[42] Guleyupoglu, B., Schestatsky, P., Edwards, D., Fregni, F. and Bikson, M. (2013) Classification of Methods in Transcranial Electrical Stimulation (tES) and Evolving Strategy from Historical Approaches to Contemporary Innovations. Journal of Neuroscience Methods, 219, 297-311.

https://doi.org/10.1016/j.jneumeth.2013.07.016 